\title{
Racial Disparity in Birth Defects: Who Has Higher Risk?
}

\author{
Ayan Ibrahim*1, Tri Tran², ${ }^{3}$, Dionka Pierce ${ }^{2}$, Julie Johnston², Nicole Richmond ${ }^{2,3}$ and \\ Susan Berry ${ }^{2,3}$
}

${ }^{1}$ Tulane School of Public Health \&Tropical Medicine, New Orleans, LA, USA; '2Louisiana DHH OPH Children and Youth with Special Health Needs Program, New Orleans, LA, USA; ${ }^{3}$ LSUHSC School of Medicine, Department of Pediatrics, New Orleans, LA, USA

\section{Objective}

This study aimed to evaluate relationships between race/ethnicity and selected major birth defects using 2006-2008 Louisiana birth defects surveillance data.

\section{Introduction}

Many studies have shown relationships between race/ethnicity and some birth defects. For example, white teenagers have higher rates of gastroschisis than African-American teenagers ${ }^{1}$, and transposition of great arteries is more prevalent in white than non-white children ${ }^{2}$. The Louisiana Birth Defects Monitoring Network (LBDMN) is a population-based surveillance system created in 2005. LBDMN actively collects information from multiple sources to track babies born with birth defects up to three years old in the state. Racial disparity in birth defects has never been evaluated using birth defects surveillance data in Louisiana. Findings from this study are expected to help LBDMN and Louisiana Children and Youth with Special Health Needs Program better understand variations in the prevalence of some birth defects among certain racial/ethnic groups in the state.

\section{Methods}

The 2006-2008linked birth defects and birth records data were analyzed. The data included only non-Hispanic white and non-Hispanic black live births whose deliveries occurred at 35 hospitals between 2007 and 2008, or at 25 hospitals during 2006. The Centers for Disease Control and Prevention (CDC) coding system, based on the British Pediatric Association and Classification of Diseases and the ICD-9-CM, was used to classify birth defects ${ }^{3}$. There were 19 birth defects with total of cases greater than 30 that were selected for analysis. Poisson regression models were used to evaluate associations between race/ethnicity and birth defects. Child's sex and gestational age, and maternal demographic and geographic characteristics were controlled for in adjusted models. SAS 9.3 was used to analyze the data and alpha was set at .05 for statistical significance.

\section{Results}

Of the total 113,696 live births included in the study, there were 3,661 cases with birth defects. Distribution of non-Hispanic white and non-Hispanic black children was $57.1 \%$ and $42.9 \%$, respectively, in the total study population. Live births that were Hispanic, Native American or Asian, which contributed to about $7.5 \%$ of total live births, were excluded from the study.

Results from adjusted Poisson regression models showed prevalence rates of the following birth defects higher in non-Hispanic white children: ventricular septal defects (prevalence ratio [PR]: 1.59, CI95\% [CI]: 1.33-1.91), atrial septal defects (PR: 1.24, CI: 1.05-1.46), patent ductus ateriosus (PR: 1.31, CI: 1.07-1.61), cleft palate without cleft lip (PR: 2.20, CI: 1.35-3.59), pyloric stenosis (PR: 2.77, CI: 1.80-4.26), obstructive genitourinary defect (PR: 1.31, CI: 1.03-1.68), gastrochisis (PR: 2.71, CI: 1.52-4.82), Down's syndrome (PR: 1.49, CI: 1.03-2.16) and hypospadias (PR: 1.50, CI: 1.21-1.86). Microcephalus (PR: 1.53, CI: 1.05, 2.24) and atrioventricular septal defects (PR: 1.67, CI: 1.01-2.76) were found to have higher prevalence rate in non-Hispanic black children.

\section{Conclusions}

Many birth defects were seen more prevalent in non-Hispanic white children than in non-Hispanic black counterparts. This difference may be results of genetic susceptibility or environmental factors which play a significant role. Further in-depth studies seeking specific genetic and environmental risk factors will be beneficial to define reasons of racial/ethnic disparity in birth defects in Louisiana.

\section{Keywords}

Birth defects; Surviellance; Data Linkage

\section{References}

[1] Williams LJ, Kucik JE, Alverson CJ, Olney RS, Correa A. 2005. Epidemiology of gastroschisis in metropolitan Atlanta, 1968 through 2000. Birth Defects Res A 73:177-183.

[2] Lorenzo DB, Correa A, Erickson JD. 2001. Racial and temporal variations in the prevalence of heart defects. Pediatrics 107(3): 1-8.

[3] Centers for Disease Control and Prevention. 2007. Metropolitan Atlanta Congenital Defects Program Defect Code List, Version 08/07. Retrieved from http://www.cdc.gov/ncbddd/birthdefects/documents/ macdpcode0807.pdf, May, 2013

\footnotetext{
*Ayan Ibrahim

E-mail: ayansaid3@gmail.com
} 